\title{
Práticas Desenvolvidas por Psicólogos em Serviços de Atenção Psicossocial: Revisão de Literatura
}

\author{
Practices Developed by Psychologists in Psychosocial Attention Services: \\ Literature Review
}

\author{
Prácticas Desarrolladas por Psicólogos en Servicios de Atención Psicosocial: \\ Revisión de Literatura
}

\author{
Stephannie Assenheimer \\ Renata Fabiana Pegoraro ${ }^{1}$ \\ Universidade Federal de Uberlândia
}

\begin{abstract}
Resumo
Este artigo tem por objetivo geral mapear as práticas dos psicólogos nos Centros de Atenção Psicossocial (CAPS) por meio de uma revisão integrativa de literatura publicada entre 2005 a 2014, em periódicos indexados nas bases de dados on-line Scielo, Biblioteca Virtual em Saúde e Redalyc, a partir das palavras-chave: Psicologia, Psicólogo, Centros de Atenção Psicossocial. A amostra foi composta por 12 artigos publicados de 2005 a 2012, cujas respostas à pergunta norteadora foram agrupadas em função das temáticas abordadas: (1) Atividades desenvolvidas pelo psicólogo nos CAPS; (2) A formação do psicólogo para o trabalho no CAPS e a necessidade de capacitação profissional; (3) Concluímos que é urgente o investimento em Educação Permanente e dos Cursos de Psicologia revejam o ensino das disciplinas sobre saúde pública.

Palavras-chave: psicólogo, Centros de Atenção Psicossocial, educação permanente, trabalho em equipe
\end{abstract}

\section{Abstract}

This article has the objective to map the practices of psychologists in Psychosocial Care Centers (CAPS). The method chosen was the integrative literature review, with articles published from 2005 to 2014, in journals indexed in online databases Scielo, Virtual Health Library and Redalyc, from keywords: psychology, psychologist, mental health services. The sample consisted of 12 articles published from 2005 to 2012 whose answers the central question were grouped according to the subjects addressed: (1) Activities developed by psychologist at CAPS; (2) The training of psychologists to work in CAPS and need for professional training; (3) Team work. We conclude that the investment in Continuing Education is urgent and the Psychology Courses need to review the teaching of the public health disciplines.

Keywords: psychologist, Psychosocial Care Centers, continuing education, team work

\section{Resumen}

Este artículo tiene el objetivo de mapear las prácticas de los psicólogos en Centros de Atención Psicosocial (CAPS). El método elegido fue la revisión integradora de la literatura, con artículos publicados desde 2005 hasta 2014, en revistas indexadas en bases de datos en línea Scielo, Biblioteca Virtual en Salud y Redalyc, a partir de palabras clave: psicología, psicólogo, servicios de salud mental. La muestra consistió en 12 artículos publicados 2005-2012 cuyas respuestas la pregunta central se agruparon de acuerdo con los temas tratados: (1) Las actividades desarrolladas por el psicólogo en el CAPS; (2) la formación de los psicólogos para trabajar en CAPS y la necesidad de la formación profesional; (3) el trabajo en equipo. Llegamos a la conclusión de que es urgente la inversión en Educación Continua y de los Cursos de Psicología revisen la enseñanza de las disciplinas sobre salud pública.

Palabras clave: psicólogo, Centros de Atención Psicosocial, la educación continua, trabajo en equipo

\footnotetext{
${ }^{1}$ Endereço de contato: Universidade Federal de Uberlândia - Rua Maranhão, s/n - Campus Umuarama, Uberlândia, MG, CEP 38405-240.
} 


\section{Introdução}

A temática deste estudo foram as práticas dos psicólogos nos Centros de Atenção Psicossocial (CAPS), um serviço de saúde mental do Sistema Único de Saúde (SUS) destinado ao cuidado de pessoas em sofrimento psíquico grave, a partir do mapeamento das publicações sob a forma de revisão integrativa de literatura. O primeiro CAPS foi inaugurado no final da década de 1980, na cidade de São Paulo, e pode ser considerado como um dos frutos da mudança da ótica com relação ao cuidado das pessoas em sofrimento psíquico grave, a partir da Reforma Psiquiátrica Brasileira (Conselho Federal de Psicologia [CFP], 2013). A inovação presente na proposta de funcionamento de um CAPS encontra-se na ruptura do modo tradicional de tratamento da psiquiatria clássica, com o cuidado alçado na corresponsabilidade da equipe multiprofissional, usuários e famílias, que em conjunto devem elaborar um plano de tratamento personalizado para cada usuário (Jucá, Medrado, Safira, Mascarenhas, \& Nascimento, 2010; Mota \& Costa, 2017).

Os CAPS são equipamentos de cuidado em contexto comunitário e devem ser pautados pelo respeito às diferenças humanas em seu ". . . processo de reelaboração do sofrimento e de 'reinvenção da vida'.' (Vasconcelos, 2004, p. 82). Recentemente os CAPS passaram a integrar a Rede de Atenção Psicossocial (RAPS) do SUS, que visa ampliar a rede de atenção a pessoas com sofrimento psíquico e que também necessitem de cuidados com relação ao uso de álcool e outras drogas (Brasil, 2011a; Belotti et al., 2017). Dados oficiais do Ministério da Saúde (Brasil, 2015) apontam 2209 unidades, as quais oferecem atendimentos individuais (ex.: farmacológico, psicoterápico) e em grupos (ex.: psicoterapia, atividades de suporte social, oficinas terapêuticas), visitas domiciliares, atendimento à família, e atividades que integrem o usuário às vivências comunitárias e familiares. Para realizar tais ações, a equipe profissional desse equipamento deve ser composta por médicos com formação em saúde mental; por enfermeiros; psicólogos, assistentes sociais, terapeutas ocupacionais, pedagogos, técnicos e/ou auxiliares de enfermagem, técnicos administrativos, técnicos educacionais e artesãos (Brasil, 2002).

Ainda que não seja obrigatória, a presença de psicólogos nas equipes do CAPS é bastante frequente. Segundo Vasconcelos (2004), isto pode ser explicado pelo fato de que ". . . são os psicólogos que possuem melhores condições para serem aprovados nos processos de seleção, dado que recebem, no curso de graduação, algum tipo de formação sistemática no campo da saúde mental" (p. 85).

De acordo com as "Referências Técnicas para atuação de Psicólogas (os) nos CAPS", elaboradas pelo CFP (2013), as atividades desenvolvidas por psicólogos devem envolver: "acoIhimento, discussão de casos em equipe, psicoterapias, atendimento às crises, elaboração de planos individuais de cuidado, grupos e oficinas, atividades dirigidas diretamente à reinserção social, dentre outras" (CFP, 2013, p. 85). Além destas, o documento ainda elenca práticas inovadoras, tais como aproximação da população rural, com a comunidade e o uso de diferentes recursos artísticos para a execução de grupos e oficinas.

Ainda segundo o CFP (2013), quase trinta mil psicólogos atuavam no SUS em 2013. Esse número expressivo aponta para a necessidade de um "redirecionamento da Psicologia, ao lado de outras profissões da saúde, em relação à sua tradição histórica relativa às orientações éticas, teóricas e metodológicas" (p. 78). Na perspectiva dos serviços alinhados à Reforma Psiquiátrica, tal como o CAPS, 
... o fato psíquico se inscreve, necessariamente, como fato social ..., [desta premissa decorre] uma nova direção de intervenção no campo da saúde mental, caracterizada pela perspectiva da atenção psicossocial, que se colocou como orientadora do desenvolvimento do projeto institucional dos CAPS. (CFP, 2013, p. 80).

Assim, podemos dizer que os psicólogos têm como desafio a possibilidade de construir a crítica ao discurso biomédico e à perspectiva reducionista acerca da experiência da loucura, que considera o sujeito apenas como um corpo enfermo, a ser contido (em hospitais psiquiátricos) e medicado. O desafio está posto como necessidade para o reconhecimento da dimensão cultural que atravessa a existência desses sujeitos e conforma suas subjetividades. Tal reconhecimento é essencial para resgatar a dimensão humana do fenômeno da loucura e a dimensão do sofrimento que atravessa essa experiência humana (CFP, 2013; Belotti et al., 2017).

Uma rápida busca efetuada na base Scielo não registrou artigo de revisão de literatura que discutissem a atuação do psicólogo no CAPS, mas, sim, artigos que problematizavam a atuação das equipes no CAPS ou a participação do psicólogo na realização de apoio matricial junto a equipes de atenção primária. Face ao que foi exposto anteriormente, o objetivo geral desta pesquisa foi mapear as práticas dos psicólogos nos Centros de Atenção Psicossocial (CAPS) voltados para o atendimento de pessoas adultas em sofrimento psíquico grave, a partir de uma revisão integrativa de literatura.

\section{Metodologia}

A pesquisa foi realizada a partir do método da revisão integrativa de literatura, que possibilita uma síntese das pesquisas relevantes sobre o assunto estudado, de forma que possa advir desta revisão uma maior sustentação para melhorias na prática de determinada área. Segundo Soares et al. (2014, p. 338) a revisão integrativa de literatura consiste em "método de reunião e síntese de resultados de investigações, originalmente construído a partir das áreas de educação e psicologia", que parte de várias perguntas ou hipóteses cuja revisão pretende responder. Além disso, este tipo de estudo aceita "integrar delineamentos de pesquisa, inclusive provenientes de diferentes paradigmas da produção do conhecimento, em nome de concretizar a complexidade do cuidado de enfermagem, que envolve integração entre questões individuais e contextuais" (Soares et al., 2014, p. 338), com grande potencial para contribuir para os cuidados em saúde.

De acordo com Mendes, Silveira e Galvão (2008), seis passos são necessários para uma revisão integrativa: (1) Identificação do tema ou questão de pesquisa; (2) Estabelecimento de critérios para inclusão e exclusão de estudos; (3) Organização das informações a serem extraídas dos estudos selecionados; (4) Análise detalhada e avaliação dos estudos incluídos na revisão; (5) Interpretação dos resultados; (6) Apresentação da revisão.

Para o presente estudo, os passos foram definidos da seguinte maneira: (1) Questão de pesquisa: "Atualmente quais as práticas realizadas pelos psicólogos nos CAPS?"; (2) Os critérios de inclusão utilizados foram: artigos publicados de 2005 a 2014, redigidos em português ou inglês, em periódicos indexados nas bases de dados on-line Scielo, Periódicos Eletrônicos em Psicologia da Biblioteca Virtual em Saúde (PEPSIC-BVS) e Redalyc, a partir das seguintes palavras-chave: Psicologia, Psicólogo, Centro(s) de Atenção Psicossocial (isoladamente 
e combinadas, neste caso com o emprego de AND). Os critérios de exclusão adotados foram: artigos redigidos em espanhol; editoriais, resumos de pesquisa, teses e dissertações; (3) Leitura dos resumos dos artigos inicialmente selecionados para avaliar se respondiam à questão norteadora; (4) Leitura dos artigos na íntegra para análise detalhada e avaliação dos estudos incluídos na revisão e extração de informações para composição de tabelas; (5) Análise e discussão dos resultados; (6) Apresentação da revisão mediante redação do artigo.

As buscas nas bases eletrônicas ocorreram no período de fevereiro a maio de 2015. A escolha da Scielo deve-se ao seu perfil de englobar periódicos de diferentes áreas do conhecimento. A Pepsic integra periódicos de Psicologia que não constam na Scielo. O Redalyc tem publicações da América Latina e Caribe. A escolha por realizar as buscas em periódicos indexados se justifica pela garantia de critérios de qualidade adotados pelas plataformas tais como a geração contínua de conteúdos (cumprimento da periodicidade e maior agilidade na avaliação dos originais recebidos) e composição do corpo editorial.

Os artigos encontrados, selecionados e, por fim, recuperados para a análise constam na Tabela 1. Partiu-se de um total de 243 artigos encontrados nas três bases eletrônicas por meio das palavras-chave. Foram inicialmente selecionados 81 pela leitura dos resumos e, após exclusão de artigos repetidos e leitura na íntegra dos artigos, foram recuperadas 12 publicações que respondiam à questão norteadora da pesquisa.

Tabela 1

Artigos selecionados nas bases on-line, excluidos e recuperados para análise.

\begin{tabular}{|c|c|c|c|c|c|}
\hline \multicolumn{6}{|c|}{ Artigos } \\
\hline 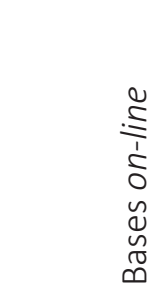 & 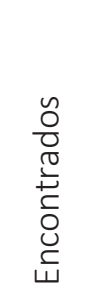 & 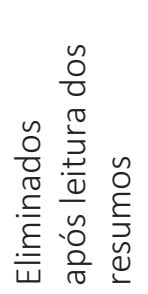 & 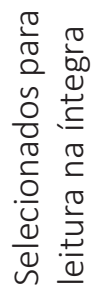 & 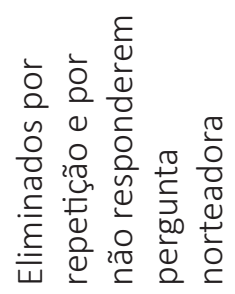 & 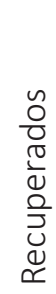 \\
\hline SCIELO & 189 & 143 & 46 & 41 & 5 \\
\hline PEPSIC & 53 & 19 & 34 & 28 & 6 \\
\hline REDALYC & 1 & 0 & 1 & 0 & 1 \\
\hline TOTAL & 243 & 162 & 81 & 69 & 12 \\
\hline
\end{tabular}

Procedeu-se à leitura na íntegra dos artigos recuperados. Dois pesquisadores independentes extraíram informações de cada uma das publicações, conforme sugerido por Broome (2000 apud Mendes et al., 2008): (a) ano de publicação; (b) fonte de publicação; (c) tipo de estudo; (d) amostra; (e) objetivos e (f) principais resultados de cada artigo. As informações foram digitadas em uma planilha eletrônica e discutidas até obtenção de consenso quanto à sua exatidão. Em seguida, foram efetuadas várias leituras de cada artigo recuperado. Os principais temas de cada um foram destacados nas leituras, e procedeu-se à classificação em temáticas, a partir dos passos elencados por Bardin (1977) para análise de conteúdo temática. Dessa etapa, emergiram três eixos de análise, descritas na seção de resultados: (1) Atividades desenvolvidas pelo psicólogo no CAPS; (2) A formação do psicólogo para o trabaIho no CAPS e a necessidade de Educação Permanente; (3) O trabalho em equipe. 


\section{Resultados}

\section{Caracterização dos Estudos}

A amostra foi composta por 12 artigos publicados de 2005 a 2012, com concentração em 2012 (cinco artigos), conforme Tabela 2. As publicações ocorreram, na maioria (nova artigos), em periódicos de Psicologia com interface com área da saúde e social. A maior parte dos artigos tinha pesquisadores de universidades públicas, e cinco publicações foram parcerias entre universidades e Secretarias de Saúde/ serviços de saúde mental. Com relação à territorialidade, encontramos seis artigos sobre CAPS situados na região Nordeste, quatro artigos contando experiências da região Sul e apenas dois artigos do Sudeste. Dentre os 12 artigos que compõem o corpus de análise, oito tinham como objetivos a compreensão da experiência ou prática profissional de psicólogos em CAPS, dois buscavam identificar o posicionamento da profissão de psicólogo frente aos serviços de atenção psicossocial, e dois procuraram conhecer o funcionamento/experiência de uma prática grupal em específico. Quanto ao tipo de pesquisa, quatro eram estudos qualitativos, um estudo exploratório, um relato de experiência profissional, um estudo quali-quantitativo, e os demais foram desenvolvidos sob a forma de análise institucional, cartografia, pesquisa-ação, pesquisa-intervenção e estudo de caso.

Tabela 2

Autores, ano de publicação, instituição de origem, objetivos, tipo de estudo e nível de evidência dos artigos recuperados ( $F=12)$.

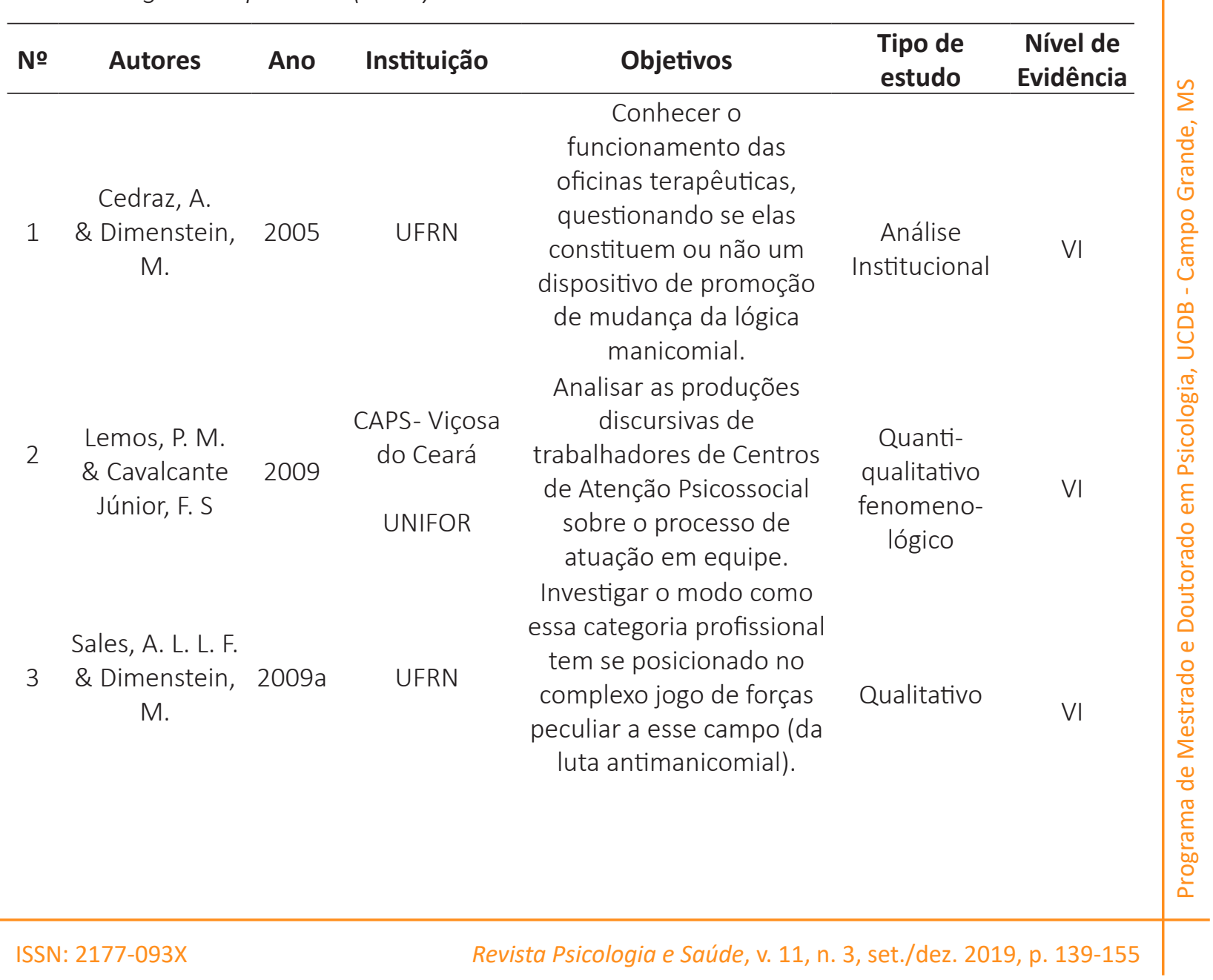


Sales, A. L .L. F.

4 \& Dimenstein, 2009b UFRN M.

\section{Campos-}

5 Brustelo, T. N, Bravo, F. F., \& Santos, M. A.

Jucá, V. J. S.,

Medrado, A.

C., Safira, L. G.,

6 Mascarenhas, 2010

L. P., \&

Nascimento,

V. G.

Paulon, S. M., Gageiro, A. M., Costa, D. F. C,

7 Londero, M. F. P, Pereira, R. G. et al.

$8 \begin{gathered}\text { Cruz, K. S., \& } \\ \text { Fernandes, A. H. }\end{gathered}$

Cantele, J.,

9 Arpini, D. M., \& 2012 Roso, $\mathrm{A}$.

$10 \begin{aligned} & \text { Lara, G. A, \& } \\ & \text { Monteiro, J. K. }\end{aligned}$

11 Larentis, C. P., \& 2012
UCS

\section{CAPS II-}

Indaiatuba, SP

USP

UFC

UNIFACS

\section{UFRGS}

Secretaria

de Estado da

Saúde do Rio

Grande do Sul

UNISINOS

UFBA

CAPS-Brusque, SC

UNISINOS

UFSM Psicologia dentro do atual modelo de atenção em saúde mental

Explorar as práticas dos psicólogos na atenção às psicoses nos CAPS do estado de Santa Catarina Investigar a organização dos CAPSad no estado do RS no que se refere às práticas e/ou intervenções psicológicas junto aos Centros de

Exame dos dispositivos clínicos que estão psicólogos, nos CAPS.
Tipo de Nível de estudo Evidência

Conhecer como tais vivenciando o cotidiano desses serviços e propõe

Cartografia por meio de entrevistas e VI observação participante

Pesquisa-ação VI

Estudo exploratório

PesquisaIntervenção sendo utilizados pelos

Estudo de Caso VI

Estudo Qualitativo (entrevistas semi estuturadas)

Qualitativa (estudo descritivo e transversal)

Estudo qualitativo revisão (descritivo e exploratório) 


\begin{tabular}{|c|c|c|c|c|c|c|}
\hline № & Autores & Ano & Instituição & Objetivos & $\begin{array}{l}\text { Tipo de } \\
\text { estudo }\end{array}$ & $\begin{array}{l}\text { Nível de } \\
\text { Evidência }\end{array}$ \\
\hline 12 & $\begin{array}{c}\text { Souza, L. G. S. \& } \\
\text { Pinheiro, L. B. }\end{array}$ & 2012 & $\begin{array}{c}\text { UFES } \\
\text { Centro de } \\
\text { Prevenção e } \\
\text { Tratamento de } \\
\text { Toxicômanos- } \\
\text { Vitória, ES } \\
\text { Unidade de } \\
\text { Saúde da } \\
\text { Família de } \\
\text { Maruípe- } \\
\text { Vitória, ES }\end{array}$ & $\begin{array}{l}\text { Relato de experiência } \\
\text { profissional construída } \\
\text { junto a usuários de um } \\
\text { CAPSad na forma de } \\
\text { Oficinas Terapêuticas }\end{array}$ & $\begin{array}{l}\text { Relato de } \\
\text { experiência } \\
\text { profissional }\end{array}$ & VI \\
\hline
\end{tabular}

Para fins de classificação de níveis de evidência, utilizaram-se os critérios propostos por Ursi (2005 apud Pompeo et al., 2009) para uma revisão integrativa. A maioria dos artigos recuperados foi classificada como nível de evidência VI, isto é, derivados de único estudo descritivo ou qualitativo, além de um único artigo de revisão bibliográfica (nível de evidência V).

\section{Eixos de Análise}

Atividades Desenvolvidas pelo Psicólogo nos CAPS

De acordo com os artigos recuperados, as três atividades mais comumente realizadas pelos psicólogos dentro dos CAPS foram: Oficinas terapêuticas (oito artigos), Acolhimento e Grupos psicoterapêuticos (ambos citados em seis artigos). Outras atividades grupais mencionadas são relacionadas a: adolescentes, família, motivação, mulheres, reencontro, relaxamento, recreativo e de orientação em saúde (antitabagismo, alcoolistas e drogadictos, prevenção de recaída), orientação profissional dos usuários, formação política e de cidadania, orientação familiar.

Além das atividades de caráter grupal, também fizeram-se presentes na prática do psicólogo, mas em um número menor de artigos: Visitas domiciliares (um artigo), Matriciamento e Acompanhamento Terapêutico (ambos citados em dois artigos). Por fim, atividades que não envolviam a assistência direta ao usuário também foram mencionadas, como: atividades relacionadas à gestão, elaboração de políticas públicas, participação em conselhos de saúde, formação de trabalhadores da saúde também foram atribuídas à profissionais da psicologia pelos artigos recuperados.

Pode-se, portanto, perceber que as práticas grupais nos CAPS contam com a presença do psicólogo. Apesar disso, apenas uma publicação, de Campos-Brustelo et al. (2010), descrevia uma intervenção grupal. Os autores relataram uma experiência com grupo de contação de histórias, o qual abria espaço para os usuários compartilharem suas histórias e incorporarem novos significados, ampliando as possibilidades de vivências.

Jucá et al. (2010) trazem que há uma grande quantidade de usuários e certa cobrança da equipe para que os psicólogos realizem grupos e oficinas nos CAPS. Esclarecem, porém, que 
as filas de espera não deveriam ser o principal motivador dessas atividades. As autoras falam também sobre um grande incômodo de os profissionais formados em Psicanálise executarem atividades de grupo, e que isso pode decorrer de um desconhecimento da produção literária sobre o tema.

Ainda que as atividades grupais tenham sido mencionadas na maior parte da literatura recuperada, Larentis e Maggi (2012) expõem que a psicoterapia individual é a prática mais comum dos psicólogos em CAPSad (mas a lógica serve para todos os outros CAPS), e que as atividades que envolvem a temática comunitária existem em escala reduzida.

Além das atividades realizadas pelos psicólogos nos CAPS, os artigos recuperados também apresentam algumas dificuldades enfrentadas pelos profissionais. Uma dessas dificuldades foi a falta de recursos materiais para atender a demanda das atividades, destacada como um empecilho para a diversidade de oficinas oferecidas (Cedraz \& Dimenstein, 2005; Sales \& Dimenstein, 2009a; Jucá et al., 2010; Lara \& Monteiro, 2012). Sobre a falta de materiais para as oficinas, as autoras Lara e Monteiro (2012) sugerem que esse problema pode ser uma oportunidade para estimular os usuários a participarem do controle social do SUS na fiscalização da aplicação dos recursos públicos.

Sales e Dimenstein (2009a) destacaram ainda como uma dificuldade a falta de profissionais qualificados para lidarem com a complexa demanda dos usuários. Já Jucá et al., (2010) trouxeram, como dificuldades para o trabalho do psicólogo nas atividades grupais, a alta rotatividade de profissionais e a dificuldade de manter sigilo em grupos terapêuticos compostos por usuários do mesmo território.

\section{A Formação do Psicólogo para o Trabalho no CAPS e a Necessidade de Educação Permanente}

O segundo eixo temático discute a formação insuficiente oferecida nos cursos de graduação para atuação na atenção psicossocial, seja por conteúdos defasados quanto ao campo da saúde pública, seja por reproduzir um modelo de consultório privado. Dois artigos apontaram conteúdos da graduação defasados (Sales, \& Dimenstein, 2009b; Paulon et al., 2011). Segundo esses artigos, os cursos de graduação em Psicologia não têm formado profissionais que atendam plenamente às necessidades dos usuários do serviço em saúde pública no contexto da Reforma Psiquiátrica. Uma hipótese para essa dificuldade seriam as grades curriculares voltadas para a formação clínica tradicional, que trata o sujeito individualmente.

O artigo de Jucá et al. (2010) investigou se a formação recebida era suficiente para atuar no CAPS. Os psicólogos que participaram do estudo dividiram-se em três grupos: (a) aqueles que se sentiam minimamente preparados pela abordagem adotada (a saber: Psicologia Analítica e Biossíntese, Gestalt-terapia, Psicanálise e Psicodrama), mas que relataram algumas dificuldades; (b) aqueles que não se sentiam preparados e, mesmo assim, tentavam atender à demanda institucional e viviam muitas angústias no processo; (c) aqueles que se recusavam a coordenar trabalhos em grupo.

Destaca-se, ainda, que a formação recebida pelos psicólogos que participaram dos estudos recuperados reproduz o modelo clínico tradicional. Nesse sentido, Paulon et al. (2011) trazem que a formação focada no modelo de clínica tradicional (privado) contribuiu para uma dificuldade em atuar na realidade da saúde pública do país, e a condução dos grupos 
por psicólogos nesses locais pode trazer um viés individualizante. Lara e Monteiro (2012) também citam que a formação nesses moldes tradicionais afeta as atuações desses profissionais no serviço da rede SUS. Uma possível solução para essa defasagem é encontrada em dois artigos que, ao discutirem a formação insuficiente dos psicólogos brasileiros, calcada em modelo clínico tradicional, destacam a necessidade de buscar formação complementar com cursos de pós-graduação (Cantele et al., 2012; Lara \& Monteiro, 2012).

De forma mais específica, Cruz e Fernandes (2012) destacam uma possível falha na graduação em Psicologia com relação ao novo modelo Antimanicomial. Segundo os autores, os profissionais podem enfrentar um engano ao apenas orientar o usuário nos caminhos que ele pode percorrer dentro da rede de atenção à saúde, e que essa atitude, por mais que seja na tentativa de ajudá-lo a compartilhar da lógica de território, pode ter uma ação contrária e inibir a sua conquista de autonomia. Essas ações tomadas pelos profissionais evidenciam que, se esses profissionais tivessem a noção de autonomia e desinstitucionalização apropriadas, suas atuações seriam diferentes.

Para além da formação recebida durante a graduação, Cedraz e Dimenstein (2005) trazem que os profissionais se queixam da falta da capacitação continuada para atuar em algumas atividades. Jucá et al. (2010) abordam que a busca pela complementação da formação profissional pode ser vista de uma maneira positiva, pois instiga o psicólogo a refletir sobre uma teoria mais próxima da prática. Contudo as autoras ressaltam que essa busca não deveria ser somente do profissional, mas de interesse das Secretarias de Saúde. Na visão dos psicólogos entrevistados por Lara e Monteiro (2012), a supervisão clínico-institucional é fundamental para sua atuação no serviço; os autores chamam a atenção, ainda, para que as supervisões institucionais possam considerar todo o conjunto institucional da rede, e que aquelas sejam espaços de compartilhamento dos sentimentos da equipe multiprofissional. Sales e Dimenstein (2009a) evidenciam que, para além da supervisão clínico-institucional, é necessário um espaço para compartilhar as inseguranças e angústias, criando um momento de cuidado para quem cuida.

Possíveis soluções para esse problema de formação são dadas pelos artigos de Sales e Dimenstein (2009a, 2009b). Para aprimorar o trabalho dos psicólogos, os autores sugerem: (1) Acionar mecanismos que possam romper com a cronificação do serviço em atenção psicossocial, mudando processos de financiamento e gestão (incluindo gestores, técnicos e usuários no processo), de ensino e de novas competências profissionais; (2) Participação em especialização para atuar em Saúde Mental, em fóruns, grupos de estudo e na construção dos próprios serviços; (3) Capacitação prévia ao início do exercício das atividades no serviço também é um ponto de destaque; (4) Interesse pessoal e a experiência profissional que, em conjunto, poderão fomentar novos modos de trabalho mais adequados à demanda do serviço.

\section{O Trabalho em Equipe}

A atuação do psicólogo em um CAPS não se dá de forma isolada. Pelo contrário, é um profissional integrado na equipe e, com relação a isso, os artigos recuperados destacaram fatores relacionados a essas equipes em sua relação com as práticas desenvolvidas pelos profissionais de Psicologia, como as reuniões de equipe, a gestão dos serviços, remuneração 
e rotatividade dos profissionais, o desafio de desenvolver ações no território, de forma intersertorial e na perspectiva da desinstitucionalização.

Sales e Dimenstein (2009a) trazem que os psicólogos entrevistados em seu estudo, muitas vezes, acabam fazendo trabalhos repetitivos, mostrando que muitas iniciativas de mudança são soterradas e acabam dando lugar à cronicidade. Estar em um CAPS geralmente demanda muito de suas emoções e expectativas, e nem sempre a remuneração está ao alcance do esforço desprendido, fazendo com que muitos se dividam entre vínculos empregatícios. Sentimentos de pavor diante de crises de usuários e dificuldades em acolher as demandas destes são indicativos de grande incômodo para esses profissionais. Além do trabalho repetitivo, Lara e Monteiro (2012) também versam sobre a rotatividade de técnicos no serviço e as relações de trabalho que podem ser perturbadas por esse fator.

Paulon et al. (2011) versam sobre as reuniões de equipe, que possuem um funcionamento burocrático, com sequência de pautas a serem discutidas, mas que não eram produtivas dentro do tempo disponível, apesar dos esforços para que as discussões fossem coletivas. As autoras chamam a atenção para o apego improdutivo a esse burocratismo, e que ele pode ser a constatação da transição do modelo manicomial para o da reforma psiquiátrica. Observam também que a equipe se queixa da falta de um momento adequado para tratar da discussão de casos nessas reuniões, e que gostariam de um espaço para construir coletivamente sobre essas demandas. Elas observaram também que os profissionais não se autorizam a reconhecer a suas ações como inovadoras, colocando-se no lugar de não saber, e que esperam uma nova teoria que responda a seus impasses cotidianos.

Um ponto interessante foi o fato de que, no artigo Cantele et al. (2012), todos os CAPS pesquisados eram coordenados por psicólogos, mostrando que esses profissionais frequentemente possuem uma posição relevante nas equipes.

Outra temática referente ao trabalho em equipe envolve o território e sobre como as equipes têm mantido (ou não) as relações com a comunidade. Cedraz e Dimenstein (2005) observaram que as oficinas do serviço pesquisado não estavam alcançando transformações em seu público-alvo, além de exigir grande parte do tempo tanto dos usuários quanto dos técnicos para sua realização, o que limitava a possibilidade de outras intervenções mais efetivas. A instituição também não possuía relações com a comunidade, ficando presa ao ostracismo. Nessa posição, ". . o o CAPS se torna, para a cidade, somente mais uma casa de loucos" (Cedraz \& Dimenstein, 2005, p. 321). Esse entendimento é encontrado também em Sales e Dimenstein (2009a) no qual os autores apontam a necessidade de os CAPS trabalharem com parcerias das mais diversas instâncias, com o objetivo de reinserir o usuário na comunidade, e atentam para o risco de produção de novas cronicidades se os serviços de assistência em saúde mental funcionarem como meros ambulatórios.

Campos-Brustelo et al. (2010) trazem que, além da equipe multidisciplinar tradicional, - CAPS pesquisado firmou parcerias com a comunidade e com voluntários, que ofereciam aos usuários "atividades de educação física, yoga, tai chi chuan, biodança, dança do ventre, grupo de leitura e grupo de apoio psicológico" (Campos-Brustelo et al., 2010, p. 3).

Lara e Monteiro (2012) relatam vários fatores negativos que têm impedido o CAPS de cumprir seu papel plenamente. A falta de clareza sobre a clientela-alvo foi um grave fator observado, além da estigmatização dos usuários e do serviço, praticadas muitas vezes pelos próprios profissionais do CAPS e da rede. O trabalho intersetorial foi citado por apenas um 
dos profissionais entrevistados. Outras dificuldades com a rede foram apontadas: a fragmentação do atendimento aos usuários e sua consequente falha, quando uma internação psiquiátrica necessária não é efetuada; e até intervenções de instituições religiosas que podem atrapalhar as ações do CAPS, onde “. . . muitos pacientes são evangélicos, isso interfere bastante, quando têm que tomar medicação, a Igreja promete que vai curar eles, e deixam de tomar medicação" (Lara \& Monteiro, 2012, pp. 87-88).

Acerca do conceito de Desinstitucionalização, que deve ser partilhado pela equipe que atua em CAPS, Cedraz e Dimenstein (2005) chamam a atenção para a forte presença de uma orientação moral dos usuários por parte dos profissionais, além da cristalização da dinâmica das oficinas, onde os usuários do serviço ocupam o lugar de observadores, sujeitos ao saber/poder dos técnicos. Os próprios usuários ficam angustiados com a eventual falta de atividades, pois elas são significadas por eles como tratamento, e, sem a sua mecânica execução para a ocupação do tempo, alguns reclamam de um sentimento de mal-estar. As autoras observaram a persistência do modelo manicomial, em que estar curado significa uma diminuição dos sintomas e da angústia, e a aceitação da medicação frequente, o que mostra entraves no entendimento do que é a desinstitucionalização. Elas concluem que as oficinas deveriam fomentar a convivência dos usuários com o diferente, estimulando formas mais criativas e autônomas de se estar no mundo, sem disciplinarizá-los como o corpo social espera.

O artigo de Sales e Dimenstein (2009a) reforça que alguns profissionais que tinham maior clareza sobre o conceito de desinstitucionalização executavam um trabalho mais próximo do que é preconizado pela Reforma. Mesmo assim, alguns psicólogos disseram reconhecer, como trabalho específico no serviço, os atendimentos individuais, o que não deixa de ser um risco para a cronificação desses profissionais.

Paulon et al. (2011) trouxeram que os profissionais entrevistados estavam questionando as práticas tradicionais prescritivas, que focam no sintoma e desconsideram o papel do usuário em seu processo de produção de saúde. O acolhimento do sujeito na sua diversidade foi um aspecto presente na concepção da equipe do serviço observado.

Para Cantele et al. (2012), além das especificações do trabalho do psicólogo, este não deve se descuidar de uma visão ampla, para permitir que esse usuário possa criar laços com o território. Lara e Monteiro (2012) também reforçam esse conceito, ao mencionar que os profissionais devem ter uma atuação política nessa sociedade, para que ela mude de postura frente à experiência da loucura.

\section{Discussão}

A maioria da amostra desta revisão vem de artigos que retratam as experiências em CAPS situados na região Nordeste com destaque para o profissional de psicologia. Em segundo lugar, aparecem as experiências da região Sul e apenas dois do Sudeste. A territorialidade da amostra vem corroborar os dados obtidos pelo Ministério da Saúde (Brasil, 2015), por ser na região Nordeste onde temos as maiores coberturas regionais e também estaduais na assistência à saúde mental (Paraíba e Sergipe). A região Sudeste começou com um patamar alto de cobertura semelhante ao das regiões Nordeste e Sul, mas agora segue com índices próximos aos das regiões Centro-Oeste e Norte, as quais enfrentam desafios para sua expansão. 
As atividades mais comumente atribuídas aos psicólogos encontradas nesta revisão foram as Oficinas terapêuticas, o Acolhimento e os Grupos terapêuticos, estando assim em concordância com o exposto nas Referências Técnicas da Profissão (CFP, 2013). É importante ressaltar que, apesar de os artigos da amostra mencionarem as práticas dos psicólogos nos CAPS, eles discorrem mais sobre os problemas na execução destas ou decorrentes dessas atividades.

Na fala dos psicólogos entrevistados nos artigos recuperados, percebe-se que o embasamento teórico das várias modalidades grupais não fornece a devida segurança para que a condução dos grupos e oficinas seja satisfatória. Isto se torna um problema ainda mais grave, pois, de acordo com as Diretrizes Curriculares Nacionais (DCN) para cursos de Psicologia elaborada pelo Ministério da Educação (MEC) (Brasil, 2011b), espera-se que o profissional dessa área tenha sua formação direcionada para a prática, a pesquisa e o ensino, sempre de forma contextualizada, isto é, levando em conta as necessidades da população-alvo do ponto de vista social e dos direitos humanos. Além disso, em seu artigo 4으, as DCN apontam que a formação do psicólogo deve auxiliar o desenvolvimento de competências e habilidades que, no caso de Atenção à saúde, formem profissionais "aptos a desenvolver ações de prevenção, promoção, proteção e reabilitação da saúde psicológica e psicossocial, tanto em nível individual quanto coletivo, bem como a realizar seus serviços dentro dos mais altos padrões de qualidade e dos princípios da ética/bioética" (Brasil, 2011b, pp. 1-2).

Assim, nas atuais DCN, a formação para atuação em práticas grupais em psicologia está prevista. No documento do Conselho Federal de Psicologia sobre a atuação nos CAPS (CFP, 2009), está atestado que as atividades grupais desenvolvidas nesse serviço tornaram-se uma das principais ações dos psicólogos, sendo que a condução dos grupos também pode ser feita em dupla, com a participação de outro profissional. Os psicólogos também podem ser responsáveis por intervenções grupais na equipe, a fim de capacitar, supervisionar e oferecer espaços de troca profissional (CFP, 2009).

Diante de todas essas leis e diretrizes resta-nos perguntar: O que tem acontecido durante a graduação que ainda não conseguimos nos adequar às normas para a formação do psicólogo e às necessidades dos serviços quanto às práticas grupais? As disciplinas sobre esta temática ainda são escassas? O conteúdo é muito generalista e não prepara para uma atuação mais incisiva? A quantidade de aulas práticas ainda é insuficiente?

O artigo de Sales e Dimenstein (2009a) retrata essa falta na formação quando destaca que alguns profissionais de Psicologia, atuantes em CAPS, demonstraram pavor diante das crises dos usuários e uma grande dificuldade em acolhê-los em suas demandas. Como já apontamos, o CAPS é um local de grande demanda emocional para os psicólogos. Isso nos leva pensar o quanto os psicólogos refletem sobre seus limites como profissionais antes de aceitarem esse trabalho que exige e que, na maioria das vezes, não paga o suficiente para que eles se dediquem exclusivamente ao serviço. Toda essa dinâmica, a nosso ver, influencia na qualidade das suas atividades nos CAPS. Concordamos, assim, com Ramminger e Brito (2011), que discorrem sobre o uso exacerbado de si nos serviços CAPS; para as autoras, "as demandas do usuário são sempre fortemente mobilizadoras, assim como as exigências formais colocadas para os serviços" (p. 154), o que implica uso da criatividade e da capacidade inventiva como uma constante. 
A necessidade de capacitação foi um tema que se repetiu em cinco artigos. É interessante pensar sobre o significado que essa palavra carrega. De acordo com o Dicionário Priberam, capacitar é "tornar-se capaz", ou seja, essa concepção de que os profissionais não chegam aptos para trabalhar em Saúde Mental mesmo após sua graduação é amplamente compartilhada pelas equipes, que demandam fortemente uma preparação inicial para adentrarem nos serviços. Dado esse significado um pouco desqualificador, preferimos aqui trocar a palavra capacitação por "Educação Permanente", uma das responsabilidades do SUS quanto à formação de recursos humanos, legalmente baseada na "Política Nacional de Educação Permanente em Saúde para o SUS", instituída pela Portaria GM/MS no 1.996, de 20 de agosto de 2007 (Brasil, 2009), e definida como:

. . . o conceito pedagógico, no setor da saúde, para efetuar relações orgânicas entre ensino e as ações e serviços, e entre docência e atenção à saúde, sendo ampliado, na Reforma Sanitária Brasileira, para as relações entre formação e gestão setorial, desenvolvimento institucional e controle social em saúde. (Brasil, 2009, p. 7).

A análise dos artigos recuperados apontou a Educação Permanente como fator importante e que tem estado em falta nos CAPS. Além de manter o profissional atualizado e mais seguro sobre a sua atuação, também é um modo de cuidar desse trabalhador em um ambiente que, muitas vezes, pode se tornar nocivo à sua própria saúde.

Dada a legitimidade dessa prática que visa ao fomento de inquietações e mudanças na atuação e nas suas concepções teóricas, ficam os angustiantes questionamentos: O que tem acontecido com o Serviço em Saúde Mental (especificamente no caso desta revisão bibliográfica, mas é um ponto a ser expandido para todo o SUS) que essas práticas não estão sendo implantadas? Os recursos são "insuficientes"? Não há interesse do Estado em cumprir as suas próprias proposições? Existem projetos histórico-políticos que não querem colocar essas diretrizes em prática? Por mais que o MEC (Brasil, 2011b) proponha que os profissionais de psicologia tenham interesse em sua própria formação continuada, como pode ser visto no trecho a seguir, é visível que somente a iniciativa pessoal não é o suficiente para capacitar os profissionais para a prática e o cotidiano dos CAPS.

Art. 4-. . . VI- Educação permanente: os profissionais devem ser capazes de aprender continuamente, tanto na sua formação, quanto na sua prática, e de ter responsabilidade e compromisso com a sua educação e o treinamento das futuras gerações de profissionais, estimulando e desenvolvendo a mobilidade acadêmica e profissional, a formação e a cooperação através de redes nacionais e internacionais. (Brasil, 2011b, p. 2)

A necessidade de educação permanente também aparece no relatório da pesquisa do CFP (2009), "Práticas Profissionais dos (as) Psicólogos (as) nos Centros de Atenção Psicossocial", onde há relatos de psicólogos que demonstram desconhecimento das políticas públicas, insegurança frente à atuação no serviço e ausência de supervisões.

Outro ponto de contato entre este estudo e o documento do CFP foi a falta de recursos materiais, humanos e espaço físico para desenvolver as atividades grupais diárias nos CAPS, de forma a poder garantir um maior número de atividades que resguardassem o sigilo das pessoas que convivem no mesmo território (CFP, 2009). 
Algumas dificuldades foram trazidas pelos artigos da amostra no que tange ao trabalho em equipe. Uma questão a ser levantada é a da cronificação do trabalho, exposto no artigo de Sales e Dimenstein (2009a). Não seria essa cronificação resultado da falta de formação? Se os profissionais não se sentem preparados para lidar com pacientes em crise, e com as diferentes demandas psicossociais que surgem, talvez fazer o que já está bem estruturado e aceito pelo saber clínico tradicional seja uma saída mais "segura". É importante ressaltar que a Reforma Psiquiátrica vem para nos tirar do lugar comum do fazer clínico-manicomial e exige da equipe novas formas de atuar diante da ciência, dos usuários, das famílias e do território.

O trabalho da equipe em um CAPS está intrinsecamente ligado à concepção de desinstitucionalização que ela partilha. Ao clarear o entendimento do que são as instituições e como elas permeiam nossas relações, mais próximos estaremos de tomar direções mais corretas no que tange às novas concepções no cuidado ao outro no campo da saúde mental. "Instituição não é uma instalação material na qual se encarnam entidades poderosas. Uma instituição é uma prática social que se repete e se legitima enquanto se repete" (Benelli \& Costa-Rosa, 2003, pp. 46-47). Não basta termos saído dos manicômios, se continuamos a carregá-los para dentro dos serviços substitutivos. "Novas instituições exigem, para sua criação, um exercício cotidiano de elaboração contínua de um projeto a partir da reflexão permanente sobre as práticas, num esforço de transformar a lógica e ação asilares" (Benelli, \& Costa-Rosa, 2003, p. 48, grifos do autor). Interessante que, no artigo de Paulon et al. (2011), isso aparece no relato das reuniões de equipe, que não eram produtivas no tempo disponível, com o apego burocráticos a pautas e questões administrativas onde não havia tempo suficiente para discussão de casos. Mesmo fora dos manicômios, o ideário enrijecido de fazer saúde mental ainda subsiste nas reuniões de CAPS, entrecortado por momentos de desinstitucionalização, em que a equipe demanda uma discussão coletiva dos casos.

\section{Considerações Finais}

O objetivo geral de mapear as práticas dos psicólogos nos CAPS foi alcançado, ainda que maior ênfase tenha sido colocada, pelos artigos recuperados, nas vicissitudes dessas práticas e do serviço CAPS.

Um limite deste estudo foi a regionalidade dos artigos, em sua maioria abordando psicólogos inseridos em CAPS da região Nordeste, seguido por Sul e Estado de São Paulo. Mas e as práticas dos profissionais psi do Centro- Oeste? E da Região Norte? E, ainda mais grave, dado o passado com forte presença de manicômios na Região Sudeste, não deveria esta região se empenhar em contar como estão sendo as suas novas práticas com relação à saúde mental?

Outra limitação do presente estudo é a impossibilidade de atribuir as deficiências na formação dos psicólogos ao currículo da graduação, pois para tal seriam necessárias pesquisas para comparar os currículos das Universidades e verificar quantas possuem disciplinas voltadas para ao entendimento de políticas públicas e a atuação profissional nos equipamentos do SUS. Para além do currículo, seria interessante que houvesse mais pesquisas feitas com alunos da graduação e egressos acerca de seus entendimentos, afetos e dificuldades encontradas em seus estudos e estágios na saúde mental e outros âmbitos do SUS. 
Cabe-nos, aqui, elencar algumas recomendações que a produção deste artigo suscitou. Como recomendações aos serviços, podemos pensar em uma maior participação dos profissionais das equipes em reuniões dos Conselhos Municipais de Saúde. Também é necessário o investimento e planejamento de ações voltadas para a Educação Permanente em Saúde. Aos Cursos de Graduação em Psicologia, é importante pensar sobre as diretrizes dos currículos novos e como eles têm afetado (ou não) a formação de seus alunos para as atividades que irão desenvolver no SUS. Por fim, à academia, destacamos a necessidade de mais pesquisas que investiguem a relação entre formação universitária e atuação profissional, campo que envolve tanto a Educação quanto a Saúde, no caso dos trabalhadores dos CAPS. Sugerimos estudos com psicólogos recém-formados pelas novas diretrizes curriculares a fim de saber se as disciplinas que dialogam com a temática de Atenção à Saúde foram fundamentais para as suas atividades no serviço, ou se ainda estão muito descontextualizadas.

\section{Referências}

Bardin, L. (1977). Análise de conteúdo. Lisboa, Portugal: Edições 70.

Benelli, S. J., \& Costa-Rosa, A. (2003). Geografia do poder em Goffman: vigilância e resistência, dominação e produção de subjetividade no hospital psiquiátrico. Estudos de Psicologia (Campinas), 20(2), 35-49. doi: https://dx.doi.org/10.1590/S0103-166X2003000200004

Belotti, M., Quintanilha, B. C., Tristão, K. G., Ribeiro Neto, P. M., \& Avellar, L. Z. (2017). Percepções sobre o processo de trabalho em um Centro de Atenção Psicossocial Infanto-Juvenil. Trends in Psychology, 25(4), 1547-1557. doi https://dx.doi.org/10.9788/ tp2017.4-04pt

Brasil. (2002). Portaria n. 336/GM, de 19 de fevereiro de 2002. Estabelece CAPS I, CAPS II, CAPS III, CAPS i II e CAPS ad II. Disponível em http://bvsms.saude.gov.br/bvs/saudelegis/ gm/2002/prt0336_19_02_2002.html

Brasil. (2009). Ministério da Saúde. Secretaria de Gestão do Trabalho e da Educação na Saúde. Departamento de Gestão da Educação em Saúde. Política Nacional de Educação Permanente em Saúde. Disponível em http://portal.anvisa.gov.br/documents/33856/396770/ ol\%C3\%ADtica+Nacional+de+Educa\%C3\%A7\%C3\%A3o+Permanente+em+Sa\%C3\%BAde/ c92db117-e170-45e7-9984-8a7cdb111faa

Brasil. Ministério da Saúde. (2011a). Portaria n. 3.088, de 23 de dezembro de 2011. Institui a Rede de Atenção Psicossocial para pessoas com sofrimento ou transtorno mental e com necessidades decorrentes do uso de crack, álcool e outras drogas, no âmbito do Sistema Único de Saúde (SUS). Disponível em http://bvsms.saude.gov.br/bvs/saudelegis/ gm/2011/prt3088_23_12_2011_rep.html.

Brasil. (2011b). Ministério da Educação. Resolução № 5, de 15 de março de 2011. Institui as Diretrizes Curriculares Nacionais para os cursos de graduação em Psicologia, estabelecendo normas para o projeto pedagógico complementar para a Formação de Professores de Psicologia. Disponível em http://portal.mec.gov.br/index.php?option=com_ docman\&view=download\&alias=7692-rces005-11-pdf\&ltemid=30192 .

Brasil. (2015). Ministério da Saúde. Saúde Mental em Dados 12. Informativo Eletrônico 10(12). Disponível em http://www.otics.org/estacoes-de-observacao/saude-mental/ acervo/arquivos/saude-mental-em-dados-12/view 
Campos-Brustelo, T. N, Bravo, F. F., \& Santos, M. A. (2010). Contando e encantando histórias de vida em um centro de atenção psicossocial. SMAD. Revista eletrônica saúde mental álcool e drogas, 6(1), 01-11. Disponível em http://pepsic.bvsalud.org/scielo. php?script=sci_arttext\&pid=S1806-69762010000100007\&lng=pt\&tlng=pt..

Cantele, J., Arpini, D. M., \& Roso, A. (2012). A Psicologia no modelo atual de atenção em saúde mental. Psicologia: Ciência e Profissão, 32(4), 910-925. doi: https://dx.doi.org/10.1590/ S1414-98932012000400011

Capacitação. (n.d.). In Dicionário Priberam da Língua Portuguesa On-line. Disponível em http://www.priberam.pt/dlpo/capacita\%C3\%A7\%C3\%A3o

Cedraz, A., \& Dimenstein, M. (2005). Oficinas terapêuticas no cenário da Reforma Psiquiátrica: modalidades desinstitucionalizantes ou não?. Revista Mal Estar e Subjetividade, 5(2), 300-327. Disponível em http://pepsic.bvsalud.org/scielo. php?script=sci_arttext\&pid=S1518-61482005000200006\&lng=pt\&tIng=pt.

Conselho Federal de Psicologia. (2009). Centro de Referência Técnica em Psicologia e Políticas Públicas. Práticas profissionais dos (as) psicólogos (as) nos centros de atenção psicossocial. Disponível em http://www.twiki.ufba.br/twiki/pub/CetadObserva/Outros/ Livro_CAPS_Psicologo.pdf.

Conselho Federal de Psicologia. (2013). Centro de Referência Técnica em Psicologia e Políticas Públicas. Referências técnicas para atuação de psicólogas (os) no CAPS - Centro de Atenção Psicossocial. Disponível em http://site.cfp.org.br/publicacao/referenciastecnicas-para-atuacao-de-psicologasos-no-caps-centro-de-atencao-psicossocial/

Cruz, K. S., \& Fernandes, A. H. (2012). Dispositivos clínicos dos psicólogos em CAPS de Salvador: Entre tutela e clínica das psicoses. Revista Psicologia e Saúde, 4(2), 94-105. Disponível em http://pepsic.bvsalud.org/scielo.php?script=sci_arttext\&pid=S2177-093X2 012000200002\&lng=pt\&tlng=pt.

Jucá, V. J. S., Medrado, A. C., Safira, L. G., Mascarenhas, L. P., \& Nascimento, V. G. (2010). Atuação psicológica e dispositivos grupais nos centros de atenção psicossocial. Mental, 8 (14), 93-113. Disponível em http://pepsic.bvsalud.org/scielo. php?script=sci_arttext\&pid=S1679-44272010000100006

Lara, G. A., \& Monteiro, J. K. (2012). Os psicólogos na atenção às psicoses nos CAPS. Arquivos Brasileiros de Psicologia, 64(3), 76-93. Disponível em http://pepsic.bvsalud.org/scielo. php?script=sci_arttext\&pid=S1809-52672012000300006\&lng=pt\&tlng=pt.

Larentis, C. P., \& Maggi, A. (2012). Centros de Atenção Psicossocial Álcool e Drogas e a Psicologia. Aletheia, (37), 121-132. Disponível em http://pepsic.bvsalud.org/scielo. php?script=sci_arttext\&pid=S1413-03942012000100009\&lng=pt\&tlng=pt.

Lemos, P. M., \& Cavalcante Júnior, F. S. (2009). Psicologia de orientação positiva: Uma proposta de intervenção no trabalho com grupos em saúde mental. Ciência \& Saúde Coletiva, 14(1), 233-242. doi: https://dx.doi.org/10.1590/S1413-81232009000100029

Mendes, K. D. S, Silveira, R. C. C. P, \& Galvão, C.M. (2008). Revisão integrativa: método de pesquisa para a incorporação de evidências na saúde e na enfermagem. Texto \& Contexto Enfermagem, 17(4), 758-764. doi: https://dx.doi.org/10.1590/S0104-07072008000400018 Mota, V. A., \& Costa, I. M. G. (2017). Relato de experiência de uma Psicóloga em um CAPS, Mato Grosso, Brasil. Psicologia: Ciência e Profissão, 37(3), 831-841. doi: https://dx.doi. org/10.1590/1982-3703004292016 
Paulon, S. M., Gageiro, A. M., Costa, D. F. C, Londero, M. F. P, Pereira, R. G., Mello, V. R. C., \& Rosa, R. H. (2011). Práticas clínicas dos profissionais 'PSI' dos Centros de Atenção Psicossocial do Vale do Rio dos Sinos. Psicologia \& Sociedade, 23(spe), 109-119. doi: https://dx.doi.org/10.1590/S0102-71822011000400014

Pompeo, D. A, Rossi, L. A, \& Galvão, C. M. (2009). Revisão integrativa: etapa inicial do processo de validação de diagnóstico de enfermagem. Acta Paulista de Enfermagem, 22(4), 434438. doi: https://dx.doi.org/10.1590/S0103-21002009000400014

Ramminger, T., \& Brito, J. C. (2011). "Cada Caps é um Caps": Uma coanálise dos recursos, meios e normas presentes nas atividades dos trabalhadores de saúde mental. Psicologia \& Sociedade, 23(spe), 150-160. doi: https://dx.doi.org/10.1590/S0102-71822011000400018

Sales, A. L. L. F., \& Dimenstein, M. (2009a). Psicologia e modos de trabalho no contexto da reforma psiquiátrica. Psicologia: Ciência e Profissão, 29(4), 812. doi: https://dx.doi. org/10.1590/S1414-98932009000400012

Sales, A. L. L. F., \& Dimenstein, M. (2009b). Psicólogos no processo de reforma psiquiátrica: práticas em desconstrução? Psicologia em Estudo, 14(2), 277-285. doi: https://dx.doi. org/10.1590/S1413-73722009000200008

Soares, C. B., Hoga, L. A. K., Peduzzi, M., Sangaleti, C., Yonekura, T., \& Silva, D. R. A. D. (2014). Revisão integrativa: conceitos e métodos utilizados na enfermagem. Revista da Escola de Enfermagem da USP, 48(2), 335-345.

Souza, L. G. S., \& Pinheiro, L. B. (2012). Oficinas terapêuticas em um Centro de Atenção Psicossocial: álcool e drogas. Aletheia, 38-39, 218-227. Disponível em http://pepsic. bvsalud.org/scielo.php?script=sci_arttext\&pid=S1413-03942012000200018\&lng=pt\&tln $\mathrm{g}=\mathrm{pt}$.

Vasconcelos, E. M. (2004). Mundos paralelos, até quando? Os psicólogos e o campo da saúde mental pública no Brasil nas duas últimas décadas. Mnemosine, 1(0), 73-90. Disponível em http://www.mnemosine.com.br/ojs/index.php/mnemosine/article/view/22

Recebido: $12 / 06 / 2017$

Última revisão: 23/05/2018

Aceite final: 31/052018

\section{Sobre as autoras:}

Stephannie Assenheimer- Psicóloga pela Universidade Federal de Uberlândia. E-mail: s.assenheimer.psico@gmail.com, Orcid: http://orcid.org/0000-0002-7401-1314

Renata Fabiana Pegoraro - Doutora e Mestre em Psicologia pela Universidade de São Paulo (USP), Ribeirão Preto, SP. Especialista em Saúde Coletiva pela Universidade Federa de São Carlos (UFSCar). Psicóloga pela USP/Ribeirão. Professora Adjunta do Instituto de Psicologia da Universidade Federal de Uberlândia (UFU). E-mail: rfpegoraro@yahoo.com.br, Orcid: http://orcid.org/0000-0001-6052-5763 
